\title{
Reducing caesarean section rate in an urban hospital serving women attending privately in India - a quality improvement initiative
}

\author{
Abhishek Bhartia* (1), Rinku Sen Gupta Dhar and Saru Bhartia
}

\begin{abstract}
Background: In line with global trends, India has witnessed a sharp rise in caesarean section (CS) deliveries, especially in the private sector. Despite the urgent need for change, there are few published examples of private hospitals that have successfully lowered their CS rates. Our hospital, serving private patients too, had a CS rate of 79\% in 2001. Care was provided by fee-for-service visiting consultant obstetricians without uniform clinical protocols and little clinical governance. Consultants attributed high CS rate to case-mix and maternal demand and showed little inclination for change. We attempted to reduce this rate with the objective of improving the quality of our care and demonstrating that CS could be safely lowered in the private urban Indian healthcare setting.
\end{abstract}

Methods: We hired full-time salaried consultants and began regular audit of CS cases. When this proved inadequate, we joined an improvement collaborative in 2011 and dedicated resources for quality improvement. We adopted practice guidelines, monitored outcomes by consultant, improved labour ward support, strengthened antenatal preparation, and moved to group practice among consultants.

Results: Guidelines ensured admissions in active labour and reduced CS (2011 to 2016) for foetal heart rate abnormalities (23 to 5\%; $p<0.001)$ and delayed progress (19 to 6\%; $p<0.001)$ in low-risk first-birth women. Antenatal preparation increased trial of labour, even among women with prior CS (28 to 79\%; $p<0.001$ ). Group practice reduced time pressure and stress, with a decline in CS (52 to 18\%; $p<0.001)$ and low-risk first-birth CS (48 to 12\%; $p<0.001)$. Similar CS rates were maintained in 2017 and 2018. Measures of perinatal harm including postpartum haemorrhage, 3rd-4th degree tears, shoulder dystocia, and Apgar $<7$ at 5 min were within acceptable ranges (13,3, 2\% and 3 per thousand respectively in 2016-18,).

Conclusions: It is feasible to substantially reduce CS rate in private healthcare setting of a middle-income country like India. Ideas such as moving to full-time attachment of consultants, joining a collaborative, improving labour ward support, providing resources for data collection, and perseverance could be adopted by other hospitals in their own journey of moving towards a medically justifiable CS rate.

Keywords: Maternity care, Caesarean section rate, Quality improvement

* Correspondence: abhishek.bhartia@sitarambhartia.org

Sitaram Bhartia Institute of Science and Research, Delhi, India

(C) The Author(s). 2020 Open Access This article is licensed under a Creative Commons Attribution 4.0 International License, which permits use, sharing, adaptation, distribution and reproduction in any medium or format, as long as you give appropriate credit to the original author(s) and the source, provide a link to the Creative Commons licence, and indicate if changes were made. The images or other third party material in this article are included in the article's Creative Commons licence, unless indicated otherwise in a credit line to the material. If material is not included in the article's Creative Commons licence and your intended use is not permitted by statutory regulation or exceeds the permitted use, you will need to obtain permission directly from the copyright holder. To view a copy of this licence, visit http://creativecommons.org/licenses/by/4.0/. The Creative Commons Public Domain Dedication waiver (http://creativecommons.org/publicdomain/zero/1.0/) applies to the data made available in this article, unless otherwise stated in a credit line to the data. 


\section{Background}

There is a global epidemic of caesarean sections (CS), which have increased from $12 \%$ in 2000 to about $21 \%$ in 2015 [1]. While many countries have a CS rate indicative of reduced access to this lifesaving procedure, the majority have a rate above the $10-15 \%$ range that is considered to be medically justifiable [2]. Even within countries there is often much variability in the CS rate based upon income level and place of delivery [3]. High CS rates are of concern because they expose the mother and child to short-term and long-term health risks [4] and impose a financial burden on families and health systems [5].

In line with global trends, the CS rate in India has increased from $8.5 \%$ in $2005-06$ to $17.2 \%$ in $2015-16$ [6]. In the same period the CS rate in private (including non-profit) facilities, which now account for more than 1 in 4 deliveries, has increased from 27.7 to $40.9 \%$ [6]. In some states the CS rate in private facilities is above 70\% [7]. While the government has laid down standards for antenatal care such as minimum of four antenatal visits, iron supplementation, haemoglobin and urine testing, and tetanus toxoid injection, private urban facilities typically have more antenatal visits, multiple routine ultrasounds and limited emphasis on antenatal education $[8,9]$. Overmedicalisation of birth as most visibly evident by the high CS rate is common.

Internationally, obstetricians' fear of litigation, convenience, and declining skills for instrumental deliveries; preference for CS by higher-income group women; feefor-service remuneration model in private healthcare; and lack of support from midwives had been identified as important factors for the rising CS rate [10]. Attempts to lower CS rate by issuing guidelines, providing uniform remuneration for both CS and vaginal deliveries, active management of labour, audit, mandatory second opinions, and expanding role of midwives had shown mixed results [10]. By the 1990s some authors had begun to raise the alarm around the issue of increasing CS in India but found few examples of hospitals that had tried similar interventions or successfully lowered their CS rate [10]. There seemed to be a widespread belief among Indian obstetricians that high rates were inevitable in private healthcare and there were no attempts by professional bodies to address this issue [10].

Our hospital in Delhi was also caught up in the caesarean epidemic with a CS rate of $79 \%$ for its 421 deliveries in 2001. We embarked on a journey to reduce this rate with the objective of improving the quality of our care and attempting to demonstrate that CS could be safely lowered in the private urban Indian healthcare setting.

\section{Methods}

\section{Local setting}

All deliveries in our 70-bed non-profit hospital, serving private patients, were conducted by fee-for-service consultant obstetricians assisted by junior obstetricians (who had recently obtained a post-graduate qualification) and nurses. The hospital had a 24-h functioning operating theatre and on-site presence of a paediatrician. All the consultant obstetricians were in solo practice and typically visited multiple hospitals. Women belonged to the upper socio-economic strata and paid for services either out-of-pocket or through private insurance. The hospital had no written clinical protocols and there was no practice of audit. Obstetricians seemed unaware of their CS rate and attributed the high rates to case-mix and maternal demand. Despite the high CS rate, women seemed satisfied with care and obstetricians showed little inclination for change. The hospital depended on consultants to bring in cases and could not insist upon a commitment for reducing CS.

A trigger for action came in the form of a formal complaint in 2001 by a woman who felt pressurised into having a prelabour caesarean for a medically unjustifiable indication.

\section{Approach}

In 2002, the chief executive hired two consultant obstetricians, who had recently completed their training, with the explicit understanding that they would work together as a unit, especially with the intent of lowering the CS rate (Table 1). They were hired full-time, on a fixed salary, to enable them to focus their attention on developing an evidence-based maternity program without financial pressures. Lacking a clear understanding of what might be a medically justifiable CS rate for our hospital we picked a figure of $25 \%$ as a goal.

The consultants started monthly review of CS cases with a senior obstetrician, developed a few clinical protocols, and started antenatal classes. Simultaneously, we started regular review of contemporary international clinical guidelines, in the hope that it would change practice and lower CS rate.

In the first year, we were able to achieve a CS rate of $40 \%$. But despite ongoing audit, visiting to other centres to observe care, attending training programmes, and inviting overseas experts to our hospital, we were unable to progress any further. With time, audit frequency decreased, and consultants began to practice individually rather than as a unit. In 2011, nine years after starting our initiative, we remained at a CS rate of above $50 \%$.

\section{Relevant changes}

Towards the middle of 2011, the chief executive decided to join an improvement collaborative as a way of refocusing on the goal of lowering CS rate and obtaining expert guidance [11]. This collaborative included several hospitals from the United States, which received monthly coaching for moving towards evidence-based 
Table 1 Sequence of initiatives introduced for reducing caesarean section (CS) rate

\begin{tabular}{|c|c|}
\hline Year & Initiatives \\
\hline 2002 & $\begin{array}{l}\text { - Created unit of full-time salaried consultant obstetricians } \\
\text { - Began monthly audit of CS cases and review of medical literature } \\
\text { - Started antenatal classes }\end{array}$ \\
\hline 2003-08 & $\begin{array}{l}\text { - Continued audit but frequency declined after } 2005 \\
\text { - Sent consultants to observe care in other maternity and midwifery units } \\
\text { - Invited obstetric expert for providing guidance on care practices }\end{array}$ \\
\hline 2011 & - Joined improvement collaborative \\
\hline 2012 & $\begin{array}{l}\text { - Began monthly presentation of data within the unit on CS and other perinatal measures with focus on low-risk } \\
\text { first birth mothers (Robson's groups } 1 \text { and 2) and with consultant name unblinded } \\
\text { - Recruited more experienced junior obstetricians for labour ward care } \\
\text { - Reached consensus on management of abnormal fetal heart rate (FHR) as per standard guidelines and started } \\
\text { regular FHR tracing review meetings }\end{array}$ \\
\hline 2013 & $\begin{array}{l}\text { - Drafted clinical guidelines for management of labour (admission, induction, augmentation, oxytocin administration, and others) } \\
\text { - Revised labour documentation to enable comprehensive data capture } \\
\text { - Trained and recruited additional nurses to enable of 1:1 support for all first-birth mothers in active labour }\end{array}$ \\
\hline 2014 & $\begin{array}{l}\text { - Drafted clinical guidelines for antenatal care } \\
\text { - Strengthened antenatal preparation with additional childbirth counsellors and involvement of birth partners } \\
\text { - Increased empowerment of junior obstetricians in the labour ward through use of structured communication } \\
\text { with consultant obstetricians }\end{array}$ \\
\hline 2015 & $\begin{array}{l}\text { - Began group practice with select consultants enabling shared decision-making for challenging cases } \\
\text { - Changed clinical leadership with "positive deviant" obstetrician becoming the lead }\end{array}$ \\
\hline 2016-2018 & $\begin{array}{l}\text { - Focused on promoting trial of labour after caesarean (TOLAC) } \\
\text { - Consolidated group practice by involving all consultants }\end{array}$ \\
\hline
\end{tabular}

maternity care and shared their progress. The collaborative used a set of interventions that had proven effective in lowering CS [12].

A team comprising the chief executive, 4 full-time salaried obstetricians, including one clinical director, and a manager with training in quality improvement was formed for this initiative.

The collaborative required detailed audit of our care protocols and clinical outcomes to identify gaps and select priority areas for improvement. The collaborative focused us on low-risk first-birth women (Nulliparous Term Single Cephalic; Robson's groups 1 and 2) as an area of maximal impact (three-fourths of $52 \%$ CS in 2011 were contributed by low-risk first-birth women). We inducted two quality officers to assist with data collection (file review and clinician interview) and analysis.

Initial analysis revealed the following deficiencies: no structured system of data collection and audit, limited collaboration among consultants, absence or poor adherence to clinical protocols, inadequate nursing support in the labour room in terms of headcount and skill, and no standard algorithms for managing indeterminate foetal heart rate (FHR) patterns or delayed progress of labour, and no standard definition of harm.

Further investigation revealed that the labour room clinical record lacked enough detail to capture all relevant information. The existing clinical protocols had been created many years before and needed to be reviewed. Nurses were rotated between the labour room and general ward resulting in sub-optimal experience with birthing. Junior obstetricians had a high turnover, as they were typically looking for opportunities for further training, and in absence of standardised care they merely followed consultant orders. Antenatal classes were offered infrequently and there weren't enough antenatal counselors in the outpatient clinic.

The labour room clinical record was revised using Plan-Do-Study-Act cycles over a period of 4 weeks. In absence of Indian guidelines, clinical guidelines from the Royal College of Obstetricians and Gynaecologists (UK), National Institute of Health and Care Excellence (UK), and the American College of Obstetricians and Gynecologists were adapted for use. Consequently, we created standard protocols for admission to the labour ward, induction, oxytocin administration, management of FHR abnormalities and other situations. All obstetricians took a formal course in electronic foetal monitoring and began regular review of FHR tracings in unit meetings.

Practice of rotating labour room nurses to the ward was stopped, additional nurses recruited, and a flexible roster introduced to ensure skilled 1:1 support for all first-time mothers in labour. Consultants developed a weekly calendar for nurse training. Junior obstetricians leaving the unit were replaced by those who had completed their training in teaching institutions and were thus more experienced and likely to have a longer tenure in the organisation.

Participation in the collaborative required monthly reporting of clinical outcomes and compliance to care practices. These measures included non-medically 
indicated delivery rate prior to 39 weeks, low-risk firstbirth caesarean rate, transfer of babies to higher level of care, and compliance with care-bundles for induction and augmentation. Figures were shared within our unit with consultant name unblinded.

Weekly meetings involving consultants, junior obstetricians, select nurses and the quality manager and officers helped identify priority areas, reach consensus on change ideas, and review impact of changes. Every month we presented our progress to different hospital teams of the collaborative and had one-on-one sessions with the collaborative faculty. Several members of the improvement team and clinical staff attended in-person meetings of the collaborative during our 2 years of enrolment.

\section{Results}

Participation in the collaborative led to transparency and understanding of our care processes and outcomes, and how these compared with best practices. Regular reporting and sharing outcomes by consultant name (within the unit) ensured sustained focus on the CS rate.

Adoption of clinical protocols, additional recruitment and training of nurses, and replacement of junior obstetricians by those with more experience reduced anxiety around management of labour. Use of structured communication by junior obstetricians promoted greater participation in clinical decision-making [13].

Compliance to guidelines for admission and oxytocin administration rose to near $100 \%$ soon after joining the collaborative. However, there was substantial variation in CS rate between consultants who continued to practice individually.

The CS rate decline in the initial 2 years (2012-13) was largely due to reduction achieved by one consultant, who attributed this improvement to antenatal sensitisation for a normal delivery, avoiding excessive weight gain, exercising to build stamina, and spending long hours with women during labour to compensate for weak nursing and junior obstetrician support. Two consultants, including the clinical director, left the unit to pursue other opportunities. The last of the original four consultants showed progress in reducing her caesarean rate 4 years (2015) after the start of the collaborative. The consultant leading the reduction in CS rate assumed leadership of the unit in 2015.

Three new consultants joining the unit in 2015-16 quickly adopted clinical protocols and commitment to promoting normal deliveries. They began group practice with shared decision-making for difficult cases, supporting each other for clinical responsibilities, and covering nights by rotation. With reduced consultant anxiety and time pressure, they became more confident about offering trial of labour after caesarean and the vaginal birth after caesarean rate improved. By the end of 2016, all consultants in the unit became part of group practice.

Between 2011 and 2016 (Fig. 1), the CS rate declined from 52 to $18 \%,(p<0.001)$. The greatest declines were for low-risk first-birth women (Robson's groups 1 and 2; 48 to $12 \%, p<0.001$; Fig. 2) and for term-single-cephalic women with previous caesareans (Robson's group 5; 90 to $31 \%, p<0.001$; Fig. 3 ). Emergency caesareans for suspected FHR abnormalities (23 to 5\% of low-risk firstbirth women, $p<0.001$ ) and delayed progress of labour (19 to $6 \%, p<0.001$; Fig. 4) also declined. Women undergoing a trial of labour after caesarean increased (28 to $79 \%, p<0.001$; Fig. 4). Elective deliveries prior to 39 weeks were almost eliminated (18\% in $2011-12$ to $1 \%$ in 2016, $p<0.001$; Fig. 5). The lower CS rates were maintained in 2017 and 2018. Measures of perinatal harm including post-partum haemorrhage, 3rd-4th degree tears, shoulder dystocia, and Apgar $<7$ at $5 \mathrm{~min}$ were within acceptable ranges $(13,3,2 \%$ and 3 per thousand in 2016-18, respectively). Women's satisfaction with care was high; $>95 \%$ gave a rating of $\geq 4$ on a 5 point scale. In contrast, the CS rate of fee-for-service

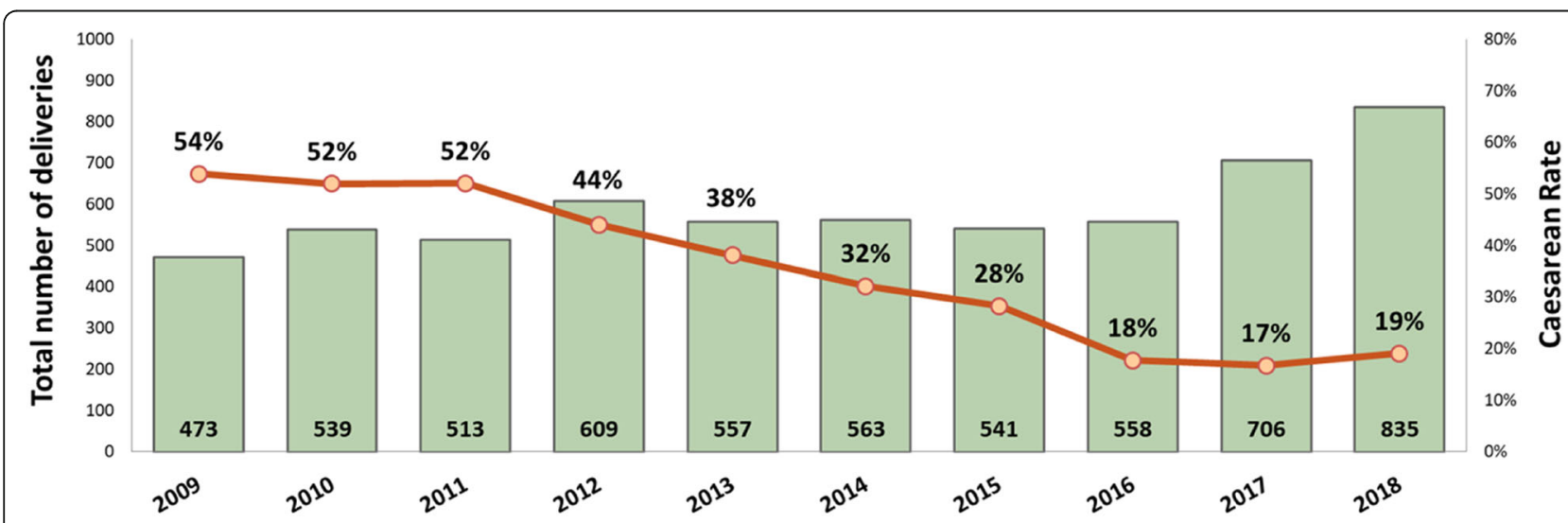

Fig. 1 Annual caesarean section rate and delivery numbers from 2011 to 2018 


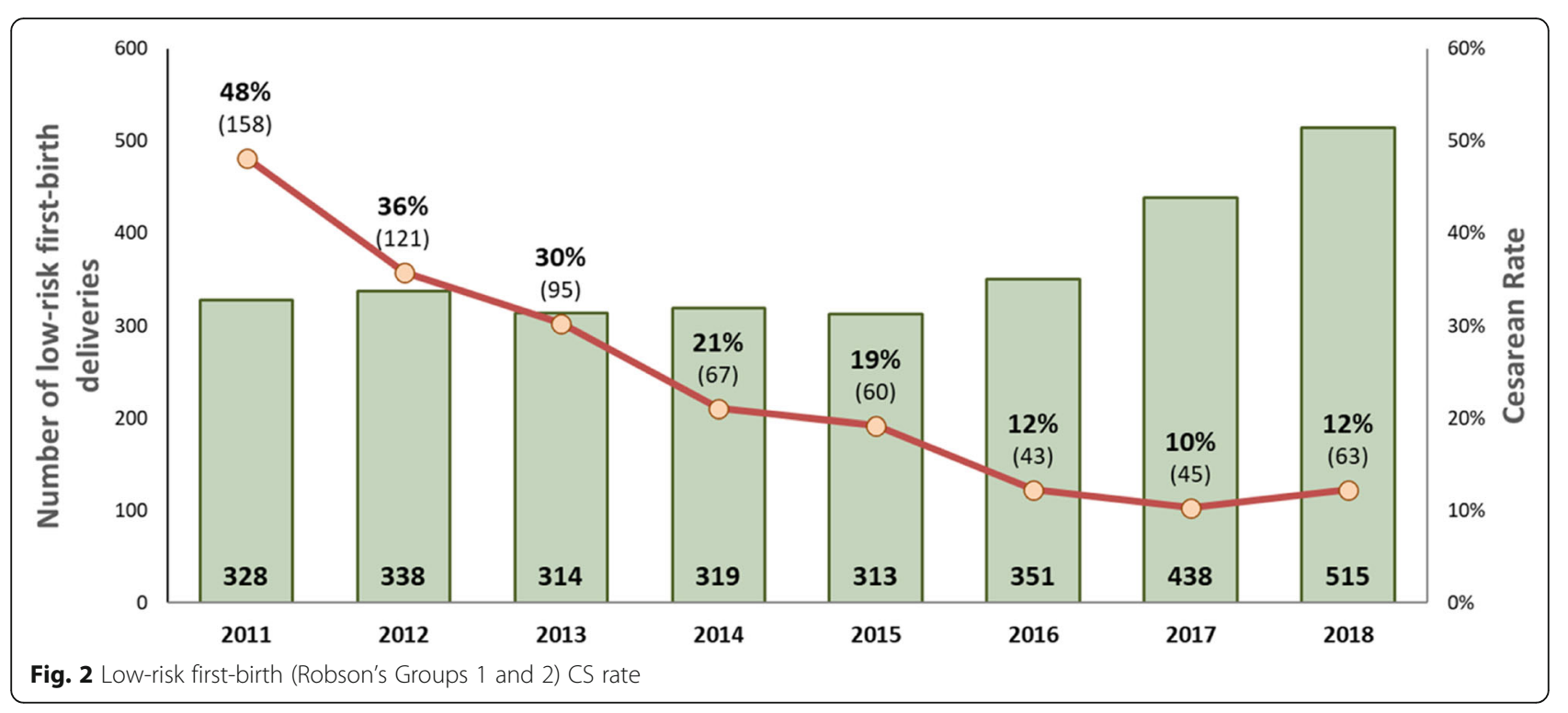

visiting consultants increased ( $p=0.02)$ marginally from 2011 (206/321; 64\%) to 2016-18 (297/412;72\%).

\section{Discussion}

To the best of our knowledge, this is a unique report of an urban hospital serving private patients in India engaging in systematic efforts for successfully lowering its caesarean rate. In comparison to an average of $54 \%$ in Delhi's private hospitals, we recorded CS rates of 1719\% during 2016-2018 [9]. Moving from a fee-forservice attachment of consultants to full-time employment changed the customer-hospital dynamic that often characterises the relationship between consultants and hospitals in private healthcare and acts as a barrier for clinical governance. Emergence of a positive deviant consultant who was able to demonstrate that lower rates were possible with "our patients, in our setting" proved critical. Other important contributors for this success included joining an improvement collaborative, hiring new medical and nursing staff, providing quality improvement support, continual close involvement of chief executive, and perseverance over 15 years.

We were able to succeed because we comprehensively addressed barriers identified by private obstetricians for lowering CS rates - maternal and family attitude, time pressure created by solo practice with attachment to multiple hospitals, inadequate labour ward support, ambiguity of clinical management due to absence of Indian practice guidelines, and lack of accountability for high CS due to absence of measurement and reporting [14]. Critically, we benefited from emergence of clinical leadership that believed in promoting normal birth and from sustained organisational commitment to this philosophy, thus shaping obstetricians' views, which are important for determining CS rate [15]. Full-time attachment at one hospital and group practice helped counter the use of CS for obstetrician convenience, another factor increasing CS [16]. Sharing outcomes by name of

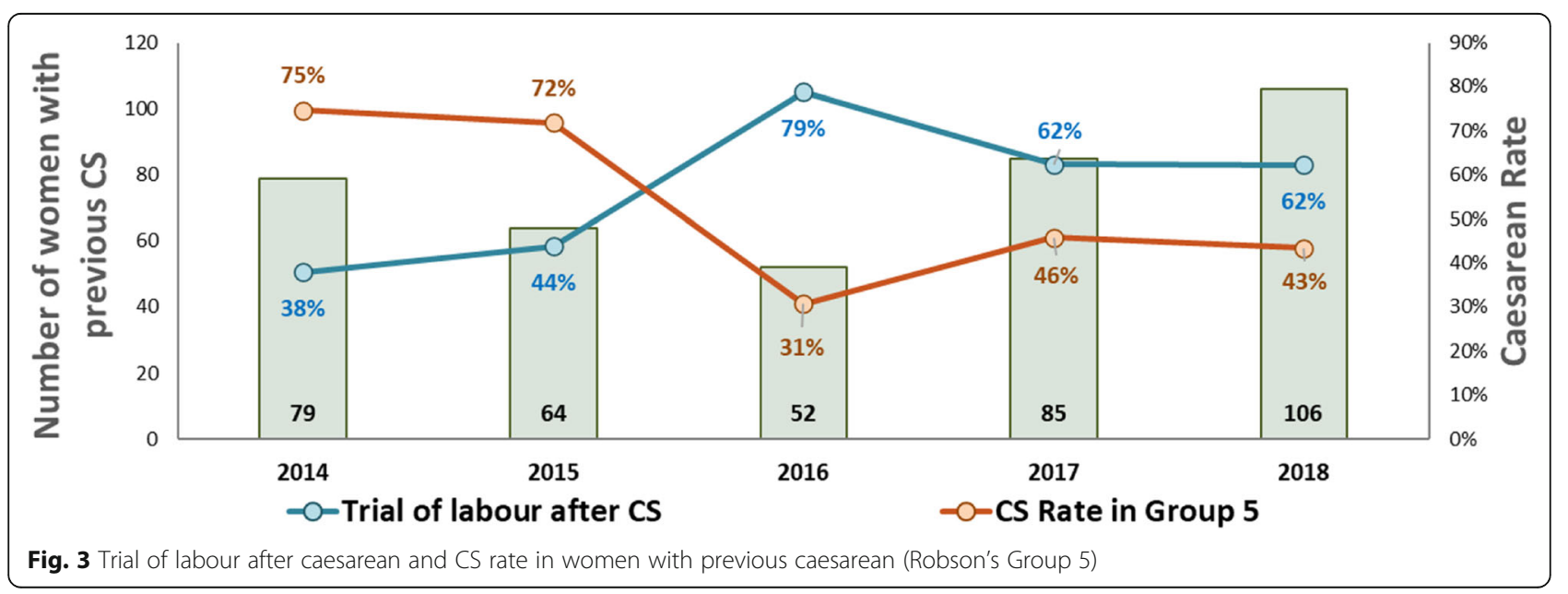




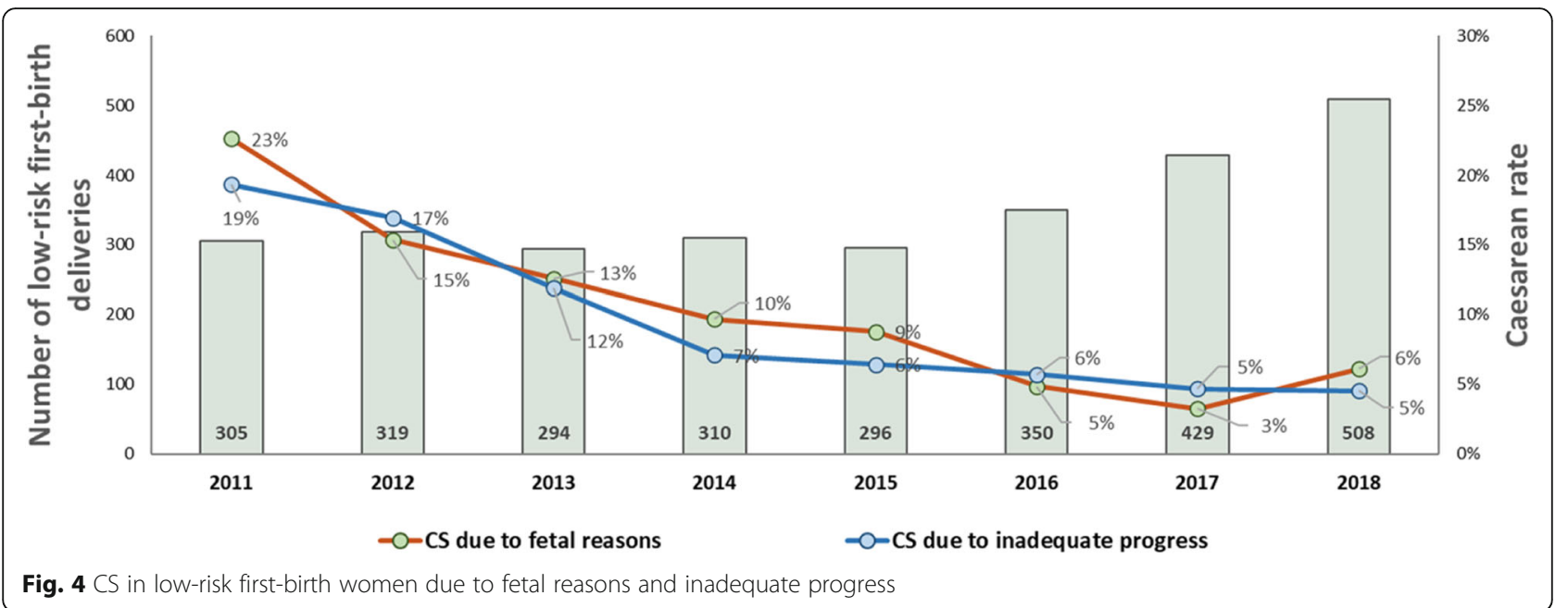

consultant, as recommended by other quality improvement collaboratives, created accountability and was likely contributor to lowering CS [17]. Despite organisational support, fear of criticism and litigation for not conducting a CS in the event of an adverse outcome remained a major concern among our consultants.

An important limitation is that our experience was not based on randomised controlled design, which compromises inferences on causality. However, the visiting feefor-service consultant comparison provides a control arm similar to a quasi-randomised study. Visiting consultants did not participate in this initiative; the marginal increase in their CS rate during the same period suggests a causal role of the intervention package. Further, with multiple overlapping interventions in an evolving context, we cannot comment on the relative contribution of various aspects.

The generalisability of our experience may be limited to relatively well-resourced private hospitals in India or other countries with similar health systems. Smaller hospitals may not be able to hire full-time consultants or increase staffing in the labour ward. However, policy changes such as requiring public disclosure of CS rate, as is now being initiated in India, might create the accountability needed to spur action [18]. Given the multiple barriers discussed above, accountability alone is unlikely to be sufficient without other enabling conditions. Introducing midwifery as an independent

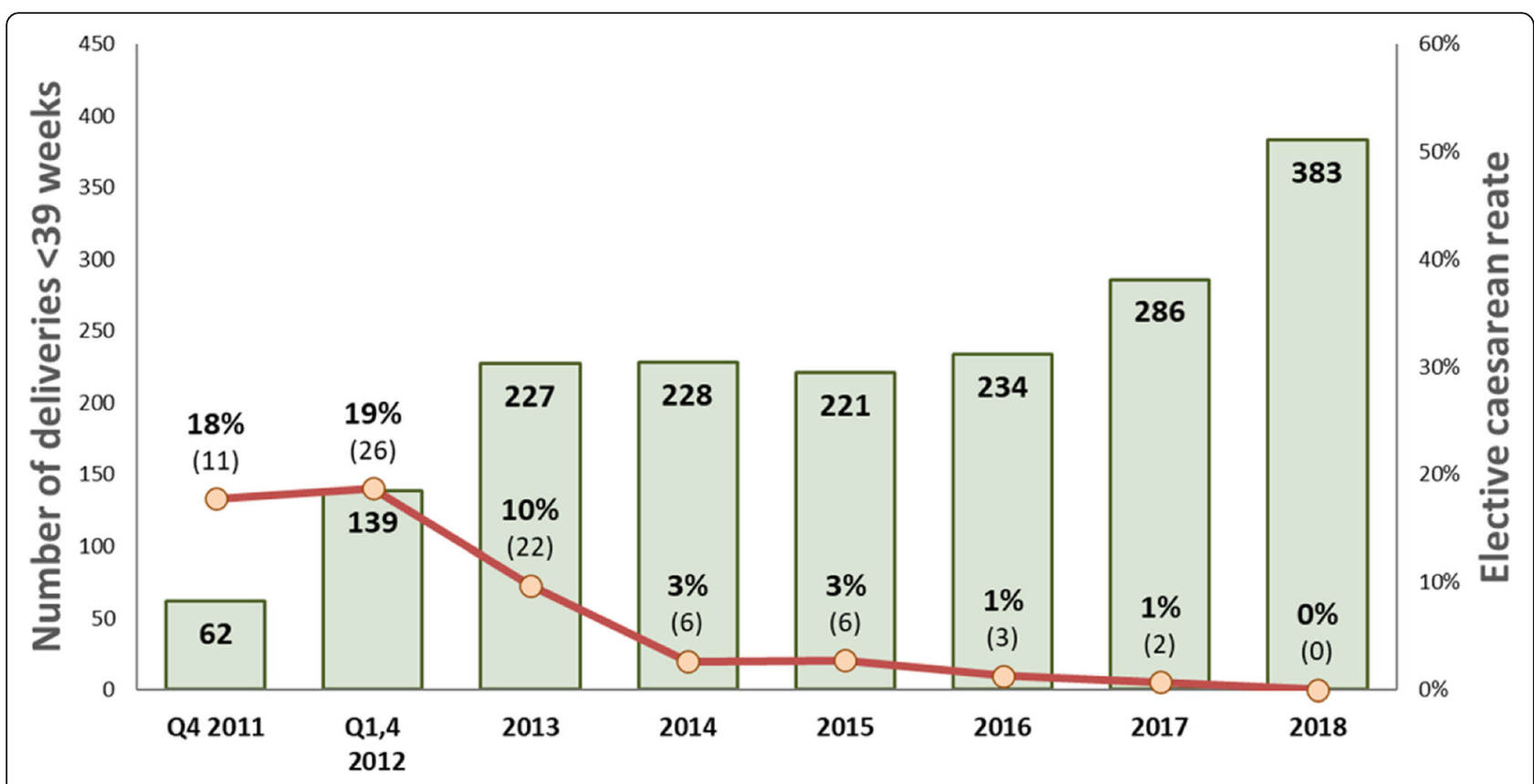

Fig. 5 Elective delivery rate prior to 39 weeks 
profession and strengthening midwifery skills of nurses who support obstetrician-led care may prove to be one of the most effective interventions for reducing CS in the long-term [19].

\section{Conclusion}

With determined systematic efforts, it is feasible to substantially reduce CS rate in private healthcare setting of a middle-income country like India where oversight of medical practice tends to be weak. Interventions such as moving to full-time attachment of consultants, joining a collaborative, improving labour ward support, providing resources for data collection, and committing to change over a long period could be adopted by other hospitals in their own journey of moving towards a medically justifiable CS rate.

\section{Abbreviations}

CS: Caesarean Section; FHR: Foetal Heart Rate; TOLAC: Trial of Labour After Caesarean

\section{Acknowledgements}

All consultant obstetricians, junior obstetricians and labour ward nurses contributed to the success of our initiative. Of particular note are the contributions of Dr. Swati Sinha and Dr. Anita Sabherwal Anand for making changes in clinical practice that helped us lower CS. Dr. Pradaya Wahi Kakkar meticulously collected and tabulated data providing us with the visibility required to keep the project progressing and for preparing this manuscript for publication. Dr. HPS Sachdev helped edit the paper for clarity and provided guidance for data analysis.

\section{Authors' contributions}

$A B$ initiated the effort to lower caesarean sections (CS) and provided ongoing management leadership. RSGD was involved as a consultant obstetrician from the very beginning and led changes in clinical practice for achieving the goal of lowering CS. SB supervised data collection and analysis and provided quality improvement support. $A B$ wrote the first draft of the paper. RSGD and SB contributed to finalising the paper. All authors have read and approved the manuscript.

\section{Funding}

No external funds were used to support the work described in this paper

\section{Availability of data and materials}

The datasets used and/or analysed during the current study are available from the corresponding author on reasonable request.

\section{Ethics approval and consent to participate}

As per the policy of the Institutional Review Board of Sitaram Bhartia Institute of Science \& Research, clinical improvement activities for aligning care with existing practice guidelines do not require ethics review. Consequently, no ethics approval or consent to participate was sought.

\section{Consent for publication}

Not Applicable.

\section{Competing interests}

The authors declare that they have no competing interests.

Received: 22 February 2020 Accepted: 3 September 2020 Published online: 23 September 2020

\section{References}

1. Boerma T, et al. Global epidemiology of use of and disparities in caesarean sections. Lancet. 2018:392:1341-8.
2. Ye J, Betrán AP, Guerrero Vela M, Souza JP, Zhang J. Searching for the optimal rate of medically necessary cesarean delivery. Birth. 2014;41:237-44.

3. Betrán AP, Temmerman M, Kingdon C, et al. Interventions to reduce unnecessary caesarean sections in healthy women and babies. Lancet. 2018 . 392:1358-68.

4. Sandall J, Tribe RM, Avery L, et al. Short-term and long-term effects of caesarean section on the health of women and children. Lancet. 2018;392 1349-57.

5. Haider MR, Rahman MM, Moinuddin M, Rahman AE, Ahmed S, Khan MM. Ever increasing caesarean section and its economic burden in Bangladesh. PLoS One. 2018:13(12):e0208623.

6. International Institute for Population Sciences. (2017). NFHS-4 (National Family Health Survey-4)-2015-16-India fact sheet. Retrieved from URL http:// rchiips.org/nfhs/pdf/NFHS4/India.pdf.

7. International Institute for Population Sciences. (2017). NFHS-4 (National Family Health Survey-4)-2015-16- state fact sheet-Telangana. Retrieved from URL http://rchiips.org/nfhs/pdf/NFHS4/TG_FactSheet.pdf.

8. Maternal Health Division, Ministry of Health and Family Welfare, Government of India (2010). Guidelines for Antenatal Care and Skilled Attendance at Birth by ANMs/LHVs/SNs. Retrieved from URL https://nhm. gov.in/images/pdf/programmes/maternal-health/guidelines/sba_guidelines_ for skilled attendance at birth.pdf.

9. Nagpal J, Sachdeva A, Sengupta Dhar R, Bhargava VL, Bhartia A. Widespread non-adherence to evidence-based maternity care guidelines: a populationbased cluster randomised household survey. BJOG. 2015;122(2):238-47.

10. Madhukar Pai. Unnecessary Medical Interventions: Caesarean Sections as a Case Study. Economic and Political Weekly, 2000;35(31):2755-61.

11. Bisognano $M$, Cherouny PH, Gullo S. Applying a science-based method to improve perinatal care: the institute for healthcare improvement perinatal improvement community. Obstet Gynecol. 2014;124:810-4.

12. Flamm B, Berwick D, Kabcenell A. Reducing cesarean section rates safely: lessons from a "breakthrough series" collaborative. Birth. 1998;25(2):117-24.

13. Shahid S, Thomas S. Situation, background, assessment, recommendation (SBAR) communication tool for handoff in health care - a narrative review. Saf Health. 2018;4:7.

14. Peel A, Bhartia A, Spicer N, Gautham M. 'If I do 10-15 normal deliveries in a month I hardly ever sleep at home.' A qualitative study of health providers' reasons for high rates of caesarean deliveries in private sector maternity care in Delhi, India. BMC Pregnancy Childbirth. 2018;18:470.

15. Ji H, Jiang $H$, Yang L, Qian $X$, Tang S. Factors contributing to the rapid rise of caesarean section: a prospective study of primiparous Chinese women in Shanghai. BMJ Open. 2015;5:e008994.

16. Murray SF. Relation between private health insurance and high rates of caesarean section in Chile: qualitative and quantitative study. BMJ. 2000;321: 1501-5.

17. Smith H, Peterson N, Lagrew D, Main E. Toolkit to support vaginal birth and reduce primary cesareans: a quality improvement toolkit. Stanford: California Maternal Quality Care Collaborative; 2016.

18. Press Information Bureau, Ministry of Health and Family Welfare, Government of India. (2018). Regulating Caesarean Sections conducted by Private Hospitals [ Press Release]. Retrieved from https://pib.gov.in/newsite/ PrintRelease.aspx? relid=177374.

19. Sandall J, Soltani H, Gates S, Shennan A, Devane D. Midwife-led continuity models versus other models of care for childbearing women. Cochrane Database Syst Rev 2016, Issue 4. Art. No.: CD004667. DOl: https://doi.org/10. 1002/14651858.CD004667.pub5.

\section{Publisher's Note}

Springer Nature remains neutral with regard to jurisdictional claims in published maps and institutional affiliations. 